\title{
Effectiveness of esophageal manometry in predicting the outcome of children with primary GER after floppy Nissen-Rossetti wrap
}

\author{
G. Mattioli, V. Gentilino, E. Caponcelli, F. Martino, M. Castagnetti, A. Pini Prato, V. Jasonni \\ Department of Pediatric Surgery, Giannina Gaslini Research Institute, University of Genova, Largo G. Gaslini 5, 16100 Genova, Italy
}

[Surg Endosc (2004) 18: 1504-1508, DOI: 10.1007/s00464-003-9307-2]

A. Pini Prato's name was misspelled in the list of authors for this article. The name is corrected here. 